\title{
Special communication
}

\section{An appraisal of gastric cancer research in cancer journals}

\author{
Nuno lunet, Rui Carvalho, and Henrique Barros \\ Department of Hygiene and Epidemiology, University of Porto Medical School, Al. Prof. Hernâni Monteiro, 4200-319 Porto, Portugal
}

\begin{abstract}
We assessed the trends in the proportion of articles on gastric cancer published in major cancer journals, the research fields of interest, and the first author's affiliation. Articles in PubMed, addressing cancer in general and stomach cancer in particular, were quantified. Abstracts of gastric cancer articles were hand-searched. The British Journal of Cancer, Cancer, Cancer Research, the International Journal of Cancer, and the Journal of the National Cancer Institute were included in the journal survey. Stomach cancer was addressed in $\mathbf{2 . 9 \%}$ of the articles in 1982-1984 and 3.3\% in 2000-2002. The proportion of articles from Asia increased (32.2\% vs 50.2\%) and that for the United States decreased (34.4\% vs $15.1 \%$ ) in 2000-2002. Articles addressing etiologic genetic factors were more frequent in $2000-2002(11.5 \%$ vs $61.6 \%)$. The proportion of stomach cancer articles was largely below the expected share considering the frequency of malignancies, and did not reflect the geography of biomedical publications. A trend was observed favoring the evaluation of genetic factors.
\end{abstract}

Key words Stomach neoplasms $\cdot$ Research $\cdot$ Trends

\section{Introduction}

The scientific, clinical, and public health priorities of the most affluent societies tend to prevail in published biomedical work, reflecting a distorted picture of the relative international burden of risk factors and disease [1]. The lower research capacity of developing countries may account for the widespread bias against health conditions that predominate in their populations [1], as less than $10 \%$ of global expenditure on health research is devoted to diseases or conditions that account for $90 \%$ of the disease burden [2]. Editorial policies, when shaped to reflect trade market values, may also be biased against diseases of poverty.

Offprint requests to: $\mathrm{N}$. Lunet

Received: March 19, 2004 / Accepted: April 7, 2004
Gastric cancer has the potential to be neglected in both research and development efforts and editorial priorities [3], because mortality has been on the decline for decades, bringing gastric cancer to a secondary setting in affluent societies, while approximately $60 \%$ of all stomach cancer cases occur in developing countries [4]. With the identification of Helicobacter pylori [5], gastric cancer was thought to be close to an end as a public health threat. However, it remains one of the most frequent malignancies worldwide (870000 new cases and 650000 deaths in 2000 [4]), with an increasing absolute number of incident cases [6], and no sound improvement observed in survival [7].

Different aspects of gastric cancer pathology and epidemiology need to be further clarified. The carcinogenesis model proposed by Correa [8] was challenged, as the two types of intestinal metaplasia may represent alternative pathways for metaplastic change, rather than successive steps of phenotypic modification of gastric mucosa. Countries with low gastric cancer rates [9] present high $H$. pylori prevalence, and a better knowledge of the worldwide variation of bacterial and human genetic polymorphisms is expected to further increase the understanding of the geographical variation in gastric cancer incidence [10-12].

Such stimulating challenges should make gastric cancer a busy research field. To assess whether the theoretical expectations have an effect in practice, we assessed the trends from 1982-1984 to 2000-2002 in terms of the proportion of articles on gastric cancer published in major cancer journals, the fields of interest in research, and the first author's affiliation.

\section{Material and methods}

American and European "large-circulation" cancer journals were selected from among those active before the discovery of $H$. pylori infection in 1984. The British 
Journal of Cancer, Cancer, Cancer Research, the International Journal of Cancer, and the Journal of the National Cancer Institute were included in the journal survey.

Through a PubMed search (www.ncbi.nih.gov/entrez/ query.fcgi) on human research for two time periods, 1982-1984 and 2000-2002, we quantified the number of articles addressing cancer as a general topic and stomach cancer specifically. The terms used for the searches were the International Standard Serial Number from each of the selected journals, and "cancer" (Medical Subject Headings and text words), to retrieve articles addressing any cancer, and "stomach cancer" or "gastric cancer", or "cardia cancer" (Medical Subject Headings and text words), to identify articles where stomach cancer was studied. A similar procedure was used for human research articles on lung, breast, colorectal and prostate cancer, and leukemia.

The abstracts of all gastric cancer articles were handsearched. When the abstract did not provide enough or clear information about its subjects or author's affiliation we screened the full paper. Articles relying exclusively on the study of cell lines or animal experimentation, referring to precursor lesions or cancers other than gastric carcinomas were discarded. Those not reporting original research were also excluded from analysis. Each of the remaining articles was classified, according to the study objective, into three categories: (i) analysis of routine data; (ii) therapy/clinical and pathological descriptions; and (iii) cancer mechanisms/ etiology/risk factors. Category (iii) was further classified according to the topics under study: Helicobacter pylori infection, diet, alcohol, tobacco, other lifestyles, genetics, or other. The first author's affiliation at publication was recorded, and countries were grouped in regions: Asia, Africa, North America, South America, Europe, and Oceania, to characterize the geography of the publications.

Proportions were compared using the $\chi^{2}$ test.

\section{Results}

With our search criteria, 14437 cancer articles were found, $3.1 \%$ addressing stomach cancer $(2.9 \%$ in $1982-$ 1984 and $3.3 \%$ in 2000-2002). The overall proportion of articles was $9.2 \%$ for lung, $18.2 \%$ for breast, $9.6 \%$ for colon and rectum, $4.0 \%$ for liver, $6.2 \%$ for prostate cancer, and $8.5 \%$ for leukemia. From 1982-1984 to 2000-2002 the proportion significantly increased for breast $(15.1 \%$ vs $19.7 \%)$, colorectal $(7.9 \%$ vs $10.5 \%)$, and prostate cancers $(2.1 \%$ vs $8.4 \%)$ and decreased for leukemia (15.2\% vs 5.0\%) (Table 1). From 19821984 to 2000-2002 the proportion of stomach cancer articles increased in the International Journal of Cancer ( $1.8 \%$ vs $5.2 \% ; P<0.01$ ), and remained similar in the British Journal of Cancer (3.0\% vs 3.9\%), Cancer (3.3\% vs $3.6 \%)$, the Journal of the National Cancer Institute $(3.1 \%$ vs $1.6 \%)$, and Cancer Research (2.1\% vs $2.0 \%)$.

In $1982-1984,37.8 \%$ of the stomach cancer articles had the first author based in North America, 32.2\% in Asia, and $25.6 \%$ in Europe. Most originated from the United States ( $n=31 ; 34.4 \%)$, Japan $(n=26 ; 28.9 \%)$, and the United Kingdom ( $n=8 ; 8.9 \%)$. In 2000-2002, North America, Asia, and Europe contributed, respectively, $15.6 \%, 50.2 \%$, and $32.0 \%$ of the articles. In the later period, the major contributors to published gastric cancer research were Japan $(n=79 ; 35.1 \%)$, followed by the United States $(n=34 ; 15.1 \%)$, and South Korea $(n=19 ; 8.4 \%)$. Regional trends were statistically significant $\left(\chi^{2}, 3\right.$ degrees of freedom $\left.=21.9 ; P<0.01\right)$.

Table 1. Proportion of articles on human cancer research addressing specific cancer sites in major cancer journals (the British Journal of Cancer, Cancer, Cancer Research, the International Journal of Cancer, and the Journal of the National Cancer Institute), and the proportion of new cancers and cancer deaths worldwide, estimated for 2000

\begin{tabular}{llclcc}
\hline & \multicolumn{2}{c}{ Articles, $n(\%)^{\mathrm{a}}$} & & \multicolumn{2}{c}{ Proportion estimated for 2000 (\%) } \\
\cline { 2 - 3 } \cline { 5 - 6 } & $1982-1984$ & $2000-2002$ & & New cancers [4] & Cancer deaths [4] \\
\hline Cancer & & & & & \\
Stomach & $141(2.9)$ & $311(3.3)$ & & 8.7 & 10.4 \\
Lung & $468(9.5)$ & $854(9.0)$ & & 12.3 & 6.0 \\
Breast & $748(15.1)$ & $1875(19.7)^{*}$ & & 10.4 & 7.9 \\
Colorectal & $392(7.9)$ & $999(10.5)^{*}$ & & 9.4 & 3.8 \\
Liver & $214(4.3)$ & $357(3.8)$ & & 5.6 & 3.3 \\
Prostate & $104(2.1)$ & $795(8.4)^{*}$ & & 5.4 & 3.1 \\
Leukemia & $752(15.2)$ & $477(5.0)^{*}$ & & 2.6 &
\end{tabular}

\footnotetext{
$* P<0.05$, proportion of cancer articles in 1982-1984 vs 2000-2002
}

a Pubmed search, as described in the "Material and methods" section 
The number of countries publishing studies on gastric cancer increased from 18 in $1982-1984$ to 24 in 2000 2002.

Approximately half the articles presented as their main objective the assessment of therapy/clinical and pathological descriptions $(68.9 \%$ in $1982-1984$ vs $40.9 \%$ in 2000-2002). Articles addressing mechanisms/ etiology/risk factors were more common in 2000-2002 compared with $1982-1984$ (28.9\% vs to $55.6 \%)$. Trends in the study aims were statistically significant $\left(\chi^{2}, 2\right.$ degrees of freedom $=20.2 ; P<0.01$ ).

In the study of mechanisms/etiology/risk factors, genetics was rarely addressed in 1982-1984 $(n=3$; $11.5 \%)$, but was the most frequent topic in 2000-2002 $(n=77 ; 61.6 \%)$. Occupation was a common topic in the first period $(n=4 ; 15.4 \%)$, but was studied in only one of the more recent studies $(0.8 \%)$. The proportion of studies including dietary factors $(19.2 \%$ vs $14.4 \% ; P=$ $0.55)$ or tobacco smoking $(3.8 \%$ vs $6.4 \% ; P=0.96)$ was similar in both periods. $H$. pylori infection was studied in $9.6 \%(n=12)$ of the articles in 2000-2002.

\section{Discussion}

In this sample of major general cancer journals, the overall proportion of stomach cancer articles was largely below its expected share if the worldwide totality of malignancies and persons affected were considered. Most articles came from Asian countries, and genetic factors have been the most common research topics in the more recent years, reflecting, at least in part, the perceived importance and novelty of the research topic.

The strategy we used to identify stomach cancer articles is highly sensitive, but lacks specificity. However, specificity was improved by hand-searching, and the analysis of publications by subject or author's affiliation included only the articles specifically addressing original human research on gastric carcinomas. In the comparisons based exclusively on the PubMed search, the lack of specificity was not confined to a particular location but related to all cancers, and we do not expect it to affect our conclusions.

The small number of articles addressing gastric cancer has a parallel in lung and liver cancers. The reason may be that stomach cancer is more frequent in developing countries, as liver cancer is today and lung cancer is expected to be in the future, following the spread of tobacco epidemics [13]. Another feature that is similar in these three cancer locations is that a high proportion of cases are attributable to only one or two agents. Up to $90 \%$ of lung cancers are attributable to cigarette smoking [14]; at least $70 \%$ of liver cancers, to hepatitis B and C virus infection [15]; and $40 \%$ to $70 \%$ of gastric cancer cases, to H. pylori infection [16]. However, interventions to decrease smoking were tested and shown to be effective [17], and a vaccine is available for hepatitis $\mathrm{B}$, but we have no vaccine against H. pylori, and the first study suggesting that eradication prevents the development of gastric cancer was published in 2004 [18].

A recent study on the global profile and trends in biomedical publication showed that, from 1995 to 2000, the publication share went slightly up for Asia, from $14.5 \%$ to $15.6 \%$, and down for North America, from $45.1 \%$ to $43.0 \%$. In the same period, the United States ranked first in the absolute number of biomedical publications per year (40.8\%), while Japan was responsible for less than 10\% [19]. Gastric cancer research does not reflect the world geography and trends in biomedical publication, as we observed an increase in the proportion of stomach cancer articles from Asian countries and a dramatic decrease in the publications originating from the United States. This under-representation of American authors was also observed in a systematic review of gastric cancer risk and genetic susceptibility, with $23 \%$ of studies attributed to the United States, and more than $50 \%$ to Asian countries [20].

The overall variation in the proportion of gastric cancer articles showed no major changes from 1982 to 2002, but large differences were observed when each of the analyzed journals was considered separately. Whether these unequal trends reflect the scientific production and its relation to the frequency of disease, lack of priority given to gastric cancer by editorial policies, or both, needs to be clarified.

In 2000-2002, a large proportion of stomach cancer articles addressed genetic factors, reflecting advances in DNA technology and human genome research, and expressing expectations for the discovery of genetic susceptibility markers and gene-environment interactions. However, the limited genetic variability and lifestyle homogeneity brought about by the large predominance of Asian studies on this subject may bias effect estimates.

Evaluation of the quality of the articles, journal editorial policies, and researchers' experience trying to publish their work will allow a better knowledge of the importance of gastric cancer in research. Meanwhile, gastric cancer ranks among the most frequent cancers in the world, but many epidemiologic features remain poorly understood, and the relative proportion of articles on these subjects seems low compared with that on most other frequent cancers.

Acknowledgment Nuno Lunet gratefully acknowledges a Grant from Fundação para a Ciência e a Tecnologia (SFRH/BD/3293/2000). 


\section{References}

1. Horton R. Medical journals: evidence of bias against the diseases of poverty. Lancet 2003;361:712-3.

2. Global Forum for Health Research. 10/90 Report on health research. Geneva: Global Forum for Health Research; 2002. Available from URL: http://www.globalforumhealth.org/pages/ index.asp. Accessed December 12, 2003.

3. Commission on Macroeconomics and Health. Macroeconomics and health: investing in health for economic development. Geneva: Commission on Macroeconomics and Health, 2001. Available from URL: http://www.cmhealth.org. Accessed December $12,2003$.

4. Ferlay J, Bray F, Pisani P, Parkin DM. GLOBOCAN 2000: Cancer incidence, mortality and prevalence worldwide, version 1.0. IARC Cancer Base No. 5. Lyon: IARC; 2001.

5. Marshall BJ, Warren JR. Unidentified curved bacilli on gastric epithelium in active chronic gastritis. Lancet 1983;I:1273-5.

6. Human cancers by organ site. Stomach Cancer. In: Stewart BW, Kleihues P, editors. World Health Organization. World cancer report. Lyon: IARC; 2003. pp 194-7.

7. Berrino F, Capocaccia R, Esteve J, Gatta G, Hakulinen T, Micheli A, et al. Survival of cancer patients in Europe: Eurocare-2 study. IARC Scientific 151. Lyon: IARC Press; 1999.

8. Correa P, Haenszel W, Cuello C, Archer M, Tannenbaum S. A model for gastric cancer epidemiology. Lancet 1975;II:58-60.

9. Lunet N, Barros H. Helicobacter pylori infection and gastric cancer: facing the enigmas. Int J Cancer 2003;106:953-60.

10. Bravo LE, van Doorn L-J, Realpe JL, Correa P. Virulenceassociated genotypes of Helicobacter pylori: do they explain the African enigma? Am J Gastroenterol 2002;97:2839-42.
11. Figueiredo C, Machado JC, Pharoah P, Seruca R, Sousa S, Carvalho R, et al. Helicobacter pylori and Interleukin 1 genotyping: an opportunity to identify high-risk individuals for gastric carcinoma. J Natl Cancer Inst 2002;94:1680-7.

12. Machado JC, Figueiredo C, Canedo P, Pharoah P, Carvalho R, Nabasis $\mathrm{S}$, et al. A proinflammatory genetic profile increases the risk for chronic atrophic gastritis and gastric carcinoma. Gastroenterology 2003;125:364-71.

13. The causes of cancer. Tobacco. In: Stewart BW, Kleihues P, editors. World Health Organization. World cancer report. Lyon: IARC; 2003. pp 22-8.

14. IARC. Tobacco smoking. IARC monographs on the evaluation of the carcinogenic risk of chemicals to humans. Vol. 38. Lyon: IARC; 1986

15. Kuper HE, Tzonou A, Kaklamani E, Hadziyannis S, Tasopoulos $\mathrm{N}$, Lagiou $\mathrm{P}$, et al. Hepatitis B and $\mathrm{C}$ viruses in the etiology of hepatocellular carcinoma; a study in Greece using thirdgeneration assays. Cancer Causes Control 2000;11:171-5.

16. Prevention and screening. Stomach cancer prevention and screening. In: Stewart BW, Kleihues P, editors. World Health Organization. World cancer report. Lyon: IARC; 2003. pp 175-8.

17. Combating the tobacco epidemic. In: World Health Organization. The world health report. Making a difference. Geneva: World Health Organization; 1999. pp 65-76.

18. Wong BC, Lam SK, Wong WM, Chen JS, Zheng TT, Feng RE, et al. Helicobacter pylori eradication to prevent gastric cancer in a high-risk region of China. A randomized controlled trial. JAMA 2004;291:187-94.

19. Rahman M, Fukui T. Biomedical publication - global profile and trend. Public Health 2003;117:274-80.

20. González CA, Sala N, Capella G. Genetic susceptibility and gastric cancer risk. Int J Cancer 2002;100:249-60. 